\section{Oncolytic virotherapy for anaplastic and poorly differentiated thyroid cancer: a promise or a clinical reality?}

\author{
Carmela Passaro ${ }^{1,2}$, Sarah Di Somma1, Anna Maria Malfitano ${ }^{1}$ \& Giuseppe Portella*,1 \\ ${ }^{1}$ Dipartimento di Scienze Mediche Traslazionali, Università degli Studi di Napoli Federico II, Napoli, Italia \\ ${ }^{2}$ Department of Neurosurgery, Brigham and Women's Hospital, Harvard Medical School, Boston, MA, USA \\ *Author for correspondence: Tel.: +39 0817463 056; Fax: +39 0817463 037; Portella@unina.it
}

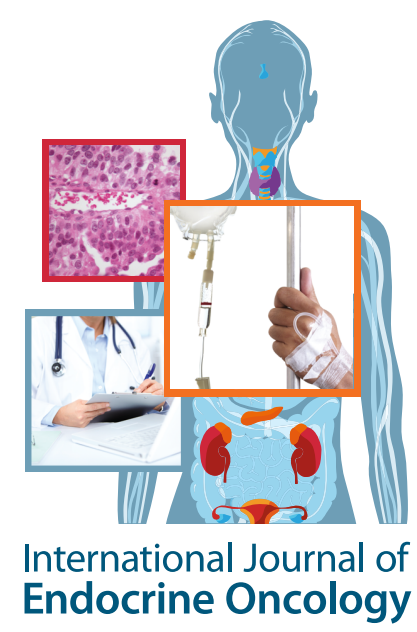

Oncolytic viruses (OVs) selectively infect and lyse cancer cells. A direct lytic effect of OVs has been theorized in the initial studies; however, the antineoplastic effect of OVs is also due to the induction of an immune response against cancer cells. Anaplastic thyroid cancer is one of the most aggressive human malignancies with a short survival time of about 6-12 months from the diagnosis. The lack of effective therapies has prompted to investigate the efficacy of OVs in anaplastic thyroid carcinoma. Different OVs have been tested in preclinical studies, either as single agents or in combinatorial treatments. In this review, the results of these studies are summarized and future perspective discussed.

First draft submitted: 10 November 2017; Accepted for publication: 21 May 2018; Published online: 1 August 2018

Keywords: anaplastic thyroid carcinoma $\bullet$ immune checkpoint inhibitors $\bullet$ immunogenic cell death $\bullet$ immunotherapy • oncolytic virus

Oncolytic viruses (OVs) are used to treat cancer due to their ability to selectively infect and lyse cancer cells, without harming normal cells or tissues [1].

Several viruses, either naturally occurring or developed through genetic engineering, are currently under investigation as oncolytic agents in clinical studies [2,3].

Currently, three OVs have been approved for cancer treatment. Rigvir, a wildtype nonpathogenic enteric cytopathic human orphan type 7 (ECHO-7) has been granted approval by the Latvian regulatory agency for the treatment of melanoma in 2004 [4]. However, this virus has not met the required regulation standard for European or US approval for clinical use.

In 2005, the oncolytic adenovirus H101 has been approved by PR China’s State Food and Drug Administration for the treatment of head and neck cancer, and, so far, a large number of patients has been treated [5].

In 2015, a herpes simplex virus (HSV) 1 expressing granulocyte-macrophage colony-stimulating factor (GMCSF) named talimogene laherparepvec (Amgen, Inc., CA, USA) was approved by the EMA (European Medicines Agency) and US FDA for the treatment of advanced melanoma [6], finally establishing OVs as a new class of cancer therapeutic agents in the US and Europe.

Thyroid malignant neoplasm is a common endocrine malignancy, most thyroid carcinomas are well-differentiated carcinomas, belonging to the papillary or follicular histotype. Papillary thyroid carcinoma comprises $80-85 \%$ of all thyroid neoplasms, follicular thyroid carcinoma accounts for approximately $10-15 \%$ of cases. Papillary thyroid carcinoma and follicular thyroid carcinoma belong to the low-risk group, have a low recurrence rate (5\%), a good prognosis and have an excellent survival rate (98\%). Poorly differentiated thyroid carcinoma (PDTC) accounts for approximately $0.5-7 \%$ of all thyroid cancers. The least common histotype is the anaplastic thyroid carcinoma (ATC), accounting for approximately $2 \%$ of all thyroid malignancies. PDTC exhibits intermediate aggressive clinical characteristics between well differentiated thyroid carcinoma and ATC, with a 5-year disease-free survival and overall survival in 43 months. PDTC are often positive for thyroglobulin. ATC has a very poor prognosis, with a mean survival time of 2-6 months for ATC. ATC occurrence is characterized by a rapid tumor growth, trachea obstruction with respiratory distress and frequent distant metastases. ATCs are negative at immunohistochemical
Future $\because$ Medicine 
analysis for thyroid transcription factor-1, and thyroglobulin. Currently, treatments for both PDTC and ATC are still challenging. Multimodal treatment, surgery, chemotherapy and radiation obtained a very limited success [7-10]. The need for novel, effective therapies of ATC has prompted the investigation of OVs efficacy in ATC experimental models since 2001 [11]. The data obtained by different groups sustain the potential clinical efficacy of this treatment. Emerging evidences of the immune-stimulatory property of OVs against tumor cells and the immunosuppressive nature of ATC microenvironment further support the clinical use of OVs for ATC treatment.

In this review we will discuss the results of studies evaluating the efficacy of OVs in ATC models and the experimental approaches aimed to increase the efficacy of OVs against ATC cells.

\section{Viruses \& cancer treatment: a 100-year-old story}

In early 1900s, studies reported cancer remissions after naturally acquired viral illnesses and the use of viruses for cancer treatment was hypothesized [12-14]. However, the lack of adequate technologies and knowledge in viral life cycle and pathogenic activity have been a hurdle for the clinical use of viruses.

Early clinical trials performed between 1950 and 1970 confirmed that viruses possessed an innate ability to kill cancer cells; however, dreadful side effects, such as hepatitis or neuroencephalitis, were also observed [12-14].

The interest in OVs was renewed in 1990s when the technological advancement together with a better understanding of the molecular alterations in cancer cells allowed manipulation of the viral genome [15] and the development of viruses selectively targeting tumor tissue with a reduced toxicity. In 1991, a landmark study reported that a modified HSV-1, with a mutation in the TK gene (TK-mutated) and with reduced neurotoxicity, replicated selectively in cancer cells and was active against glioma in mice brains [16].

In same years, another OV has been engineered: $d l 1520$ (ONYX-015). $d l 1520$ is an oncolytic adenovirus that carries the deletion of the $E 1 B 55 K$ gene. The lack of E1B55K product makes the virus defective in $\mathrm{p} 53$ binding/degradation and nuclear export of viral mRNA, functions that are essential for viral propagation in normal cells $[17,18]$. In the great majority of cancer cells, the $E 1 B 55 K$-deletion is complemented by functional inactivation of $\mathrm{p} 53$ and alterations in mRNA export mechanisms. E1B55K is required for other important functions, and it has been observed that its deletion greatly impairs viral replication explaining the modest activity as single agent reported in clinical trials [18].

$d l 1520$ was the first virus to enter Phase I clinical trials. Eighteen Phase I and II clinical trials were performed with $d l 1520$ and safety was demonstrated with all routes of administration [19]. No Phase III clinical trial has ever been completed, due to legal issues, but in $2005 \mathrm{H} 101$ an adenovirus with the same deletion of $d l 1520$ was licensed in PR China for the treatment of head and neck cancers [5]. The approval was based on the results of a Phase III trial, with a $79 \%$ response rate in patients receiving $\mathrm{H} 101$ by intratumor administration in combination with cisplatin and 5 -fluorouracil, while a $40 \%$ response rate was reported in control studies without virus treatment $[5,20]$.

Overall, the clinical results obtained with $d l 1520$ and H101 have been pivotal in demonstrating the safety profile of OVs, and paving the way for the development of more effective OVs.

Novel and more effective OVs were developed, employing different strategies and viral strains. In 2015, an HSV1 expressing GM-CSF called talimogene laherparepvec has been approved by EMA and FDA for the treatment of advanced melanoma [6]. Other OVs (https://clinicaltrials.gov/ClinicalTrials.gov Identifiers: NCT03206073, NCT03294486, NCT03225989) are currently under clinical evaluation and the number of OVs approved for cancer treatment is expected to increase in the near future.

Recently, the interest toward OVs has been increased by observations showing that OVs exert potent immunostimulatory effects against tumor cells, and nowadays OVs are considered as immunotherapeutic agents [21]. The rational combination of OVs with immunotherapeutic checkpoint inhibitors is another relevant pro for their clinical use [22].

\section{Tumor selectivity of OVs}

OVs can be divided into two broad groups: OVs with a natural tropism toward cancer cells and OVs genetically engineered to selectively replicate in cancer cells. For both groups the deficiency in antiviral response in cancer cells is a crucial mechanism of tumor selectivity. In normal cells viral infection elicits the release of IFNs, produced following the detection of viral RNA, DNA or proteins by intracellular pathogen recognition receptors. This signaling pathway results in the expression of hundreds of effector genes known as IFN-stimulated genes. These genes are the basis for an elaborate effector mechanism aiming to the clearance of the viral infection [23,24]. Moreover, TLR activation due to the recognition of viral elements activate downstream pathways, leading to PKR 
activation [23]. Phosphorylated PKR blocks protein synthesis preventing viral replication. Neoplastic cells have been shown to have an abnormal IFN pathways, and/or abnormal PKR activity, making them more susceptible to viral infection [24,25].

\section{Selective OVs in thyroid cancer models}

\section{OVs with natural selectivity}

Some viruses have a natural selectivity toward cancer cells (natural OVs), exploiting the aberrant signaling pathways that sustain cancer growth. For instance, constitutively active AKT pathway signaling serves as a sustained growth and survival signal in many different types of cancer [26]. Wang and colleagues demonstrated that the natural tropism of myxoma virus in cancer cells capitalizes on endogenous AKT activity via complex formation between AKT and M-T5, a myxoma viral protein [26]. A myxoma virus MYXV has been tested in a preclinical study for the treatment of ATC, although with a limited success in cell killing and in productive infection of ATC cells [27].

Vaccinia virus (VACV) shows a natural selectivity for tumors. The abnormal vascular structure of tumors likely represents the major determinant of the tropism [28]. Further selectivity for VACV can be achieved through the deletion of the $T K$ gene, involved in nucleotide synthesis, limiting viral replication to nucleotide rich cancer cells $[29,30]$. Among VACVs, the most effective OV is JX-963. This virus has been engineered to target cancer cells presenting activation of the transcription factor E2F and the EGFR pathway, by deletion of the TK and vaccinia growth factor genes. It also expresses the human GM-CSF.

JX-963 has already been tested in a wide range of human cancer cell lines and in clinical trials [31].

GLV-1h68 is a replication-competent VACV targeting tumor cells. It bears mutations in two loci: $F 14.5 \mathrm{~L}$, $J 2 R$ (encoding thymidine kinase) and $A 56 R$ (encoding hemagglutinin). This virus also carries marker genes (Renilla luciferase, GFP and $\beta$-gal) that allow imaging of tumor-specific entry and replication [32]. GLV-1h68 has been tested in ATC cells and tumor xenografts, showing a significant inhibition of tumor growth after a single administration $[33,34]$.

A variant of GLV-1h68 carrying hNIS symporter gene was developed showing re-expression of hNIS in ATC cells [35]. hNIS expression could be useful both as a marker gene for the tracking of the virus and as a potential sensitizer to radiotherapy using Iodine-131. A recombinant variant of tanapoxvirus has been used as OV. Tanapoxvirus is an attractive candidate for virotherapy, infected patients experience a mild and self-limiting febrile illness, no transmission from person to person has been observed [36]. Also for tanapoxvirus, ablation of the viral thymidine kinase has been used as a strategy to increase cancer cell selectivity. A tanapoxvirus has been already tested in studies involving thyroid cancer cells [27].

Other natural OVs include oncolytic attenuated measles virus (MV) [37], Mumps virus [38] and Newcastle Disease Virus [39]. The natural selectivity of these viruses might be related to the overexpression of surface molecules acting as viral receptors on cancer cells. Wild type and attenuated MVs have different receptors; attenuated MVs have a specific tropism for CD46 receptor. CD46 receptor protects cells from complement attack blocking the complement cascade and serving as co-factor for inactivation of $\mathrm{C} 3 \mathrm{~b}$ and $\mathrm{C} 4 \mathrm{~b}$, thereby protecting neoplastic cells from complement mediated lysis [40]. Overexpression of CD46 has been observed in most human cancers including thyroid carcinomas [41].

In cells showing a high expression of CD46 receptor, common feature of cancer cells, infection with attenuated MV leads to an extensive intercellular fusion (cytopathic effect) resulting in the killing of cancer cells. Interestingly, levels of CD46 in cancer cells can predict the efficacy of oncolytic treatment with MV [42].

An attenuated MV, vaccine strain MVEdm, has been used in studies with thyroid carcinoma models. This virus is active against a broad range of human cancer cells and has been modified to include the NIS gene (MV-NIS), to allow infected cells to transport iodide ions into the cell. The inclusion of the NIS gene in the viral construct will render radioiodine-resistant ATC susceptible to radioiodine therapy.

In vitro and in vivo experiments demonstrated the oncolytic efficacy of MV-NIS in ATC-derived cell lines. Radioactive iodine uptake along with single-photon emission computed tomography imaging of xenografts confirmed NIS expression both in vitro and in vivo. Administration of radioiodine, to MV-NIS-treated KTC-3 tumors, showed an increased tumor cell killing [43].

Oncolytic parvovirus selective activity against human cancer cells relies on the intrinsic preference of this virus for proliferating and metabolically altered cancer cells. The oncoselectivity of parvovirus is a complex phenomenon based in part on multiple tumor cell-specific determinants. Molecular pathways involved in $\mathrm{H}-1$ parvovirus ( $\mathrm{H}-$ $1 \mathrm{PV})$ selective tumor targeting are described elsewhere [44,45]. The oncolytic H-1PV have not been tested yet in 
thyroid models. However, studies using H-1PV could be useful not only to test its efficacy against thyroid cancer cells, but also to evaluate the interactions between immune response and viral treatment in thyroid carcinomas. Indeed, the lack of immune competent animal models has prevented a better understanding of these interactions. The natural host of $\mathrm{H}-1 \mathrm{PV}$ is the rat and the virus is effective against rat tumor cells. Rat thyroid cell lines transformed with oncogenes involved in thyroid carcinogenesis and tumorigenic in syngeneic animals are available together with rat models of poorly differentiated and metastatic thyroid carcinoma [46-48]. These experimental models could be useful to analyze the immune stimulatory functions of H-1PV.

\section{Tumor selectivity by viral genome modifications}

Genome modification has been an important strategy to obtain a selective viral infection/replication in tumor cells. Two major strategies were exploited: re-targeting the selectivity of the viruses, and controlling the selectivity of replication via viral gene inactivation, transcriptional targeting, microRNA targeting sequences $[1,49,50]$.

Here, we will discuss only the strategies regarding viruses used in studies focused on thyroid carcinomas.

The oncolytic adenovirus $d l 1520$, bearing a $E 1 B 55 K$ gene deletion, was the first OV used in thyroid carcinoma cell lines and ATC xenografts [11]. $d l 1520$ showed to be active as single agent; however, its efficacy was increased when used in combination with doxorubicin, paclitaxel, ionizing radiation and lovastatin [51,52]. The results obtained in these preclinical studies have demonstrated the potential efficacy of $d l 1520$ in combination with antineoplastic agents for the treatment of ATC.

The experience acquired with $d l 1520$ led to the development of oncolytic adenoviruses with small specific gene deletions to retain both viral potency and tumor selectivity. By deleting the E1ACR2 domain ( 24 amino acids), viral replication can only proceed in cells with deregulated growth control, with altered G1/S checkpoint and $\mathrm{pRb} / \mathrm{Ras}$ signaling [53], found in the majority of cancer cells [54]. dl922-947, an adenoviral mutant with the E1ACR2 deletion, showed a significantly higher efficacy than $E 1 B 55 K$-deleted variants.

dl922-947 has been extensively studied in thyroid carcinoma models alone or in combination with other agents, in other words, anti VEGF humanized antibody bevacizumab, ionizing radiation and other agents [55-58].

A conditionally replicative adenovirus (named 'HILMI'), replicating specifically in cells with an active Wnt/ $\beta$ catenin pathway, was also developed.

HILMI replicates in cells by virtue of TCF response elements driving the expression of adenoviral genes $E 1 \mathrm{~A}$ and $E 1 B$. A fraction of undifferentiated or poorly differentiated thyroid cancers contain mutations in $\beta$-catenin gene. Thyroid cancer cell lines, derived from undifferentiated or anaplastic carcinomas and with an active Wnt $/ \beta$ catenin pathway, were efficiently killed by HILMI; indeed, prolonged survival of mice with ATC tumors was observed following HILMI administration [59].

ONYX-411 is a conditionally replicative oncolytic adenovirus tested in thyroid cancer experimental models. In particular, the expression of the E1A and E4 genes, are under the control of the human E2F promoter, as E2F levels are high in tumor cells, this promotes viral replication. To prevent adenoviral-induced cell cycle progression in normal cells, the E1A gene of ONYX-411 was modified by deleting CR2 domain impairing pRB binding. ONYX-411 was shown to target and destroy ATC cell lines and reduced the growth of xenograft tumors in vivo.

Adenoviral mutant dl309 lacking several E3 region genes dl309 was tested in human K1 cells derived spontaneously from a papillary thyroid carcinoma. E3 region proteins have a prominent role in immune evasion and prevent innate immunity. $d l 309$ has enhanced cytopathogenicity compared with Ad5wt in range of human and mouse cells. To better establish viruses in inducing cell death, tumor-derived cell lines that have not undergone extensive passage were used. The K1 cell line are non-tumorigenic, contact-inhibited and retain DNA damage checkpoints [60]. Authors observed high levels of apoptosis in $\mathrm{K} 1$ infected cells likely correlated to the expression of viral gene E1A. However, dl309 virus has not been tested against tumorigenic ATC cell lines nor was tested its efficacy to stimulate an innate or acquired immune response against cancer cells.

Different OVs other than adenoviruses, were tested in thyroid carcinoma models. G207 is a conditionallyreplicative HSV-1 with ICP34.5 deletion and UL39 disruption [61-63]. ICP34.5 is a neurovirulent factor that induces the dephosphorylation of the translation initiation factor eIF-2A, ultimately counteracting the host shutoff of protein synthesis. The UL39 gene encodes for the large subunit of the ribonucleotide reductase (ICP6) required for viral DNA synthesis [64]. The selectivity is due to higher levels in cancer cells of the ribonucleotide reductase (an ICP6 cellular homolog that could provide the missing function) [65].

G207, combined with paclitaxel, showed synergistic cytotoxicity against ATC cells [66].

A variant of G207 expressing Escherichia coli LacZ (NV1023) has been also tested in thyroid models [67]. 


\begin{tabular}{|c|c|c|c|c|}
\hline Virus family & Virus type & ATC cell line & Xenograft study & Ref. \\
\hline \multirow[t]{4}{*}{ Poxvirus } & $\begin{array}{l}\text { Vaccinia virus } \\
\text { Myxoma virus } \\
\text { Tanapox virus }\end{array}$ & $\begin{array}{l}\text { SW1736, U-HTh7, } \\
\text { C643, THJ-11T, } \\
\text { THJ-16T, THJ-21T, } \\
\text { and THJ-29T, } \\
8505 C \text {, ASH3, } \\
\text { KMH2, BHT-101, Cal } 62\end{array}$ & No & [36] \\
\hline & GLV-1h68 & $\begin{array}{l}\text { 8505C, 8305C, } \\
\text { KAT4C, KAT4, } \\
\text { KAT18, DRO90-1 }\end{array}$ & No & [42] \\
\hline & GLV-1h68 & $8505 C$, DRO90-1 & Yes & [43] \\
\hline & GLV-1h153 & $8505 C, 8305 C$, FRO & Yes & [44] \\
\hline \multirow[t]{6}{*}{ Adenovirus } & ONYX-015 & ARO, KAT4, FRO & Yes in combination with radiotherapy & {$[14,69]$} \\
\hline & ONYX-015 & ARO, KAT4, FRO, Cal62 & Yes in combination with lovastatin & [60] \\
\hline & $d / 922-947$ & ARO, FRO, KAT4 & Yes in combination with bevacizumab & [70] \\
\hline & $d 1922-947$ & BHT-101-5, FRO, Cal62 & Yes in combination with ionizing radiation & [71] \\
\hline & dl922-947 & BHT-101-5, FRO, Cal62. $8505 \mathrm{C}$ & Yes in combination with $A_{Z Z D_{1152}}$ & [72] \\
\hline & dl922-947 & BHT-101-5, FRO, Cal62 & Yes in combination with olaparib & [73] \\
\hline \multirow[t]{3}{*}{ Herpes virus } & NV1023 & DRO90-1, ARO, KAT-4C, KAT- 18 & Yes & [74] \\
\hline & G47 & ARO, FRO & Yes & [75] \\
\hline & G207 & DRO90-1, KAT-4 & Yes in combination with paclitaxel & [76] \\
\hline
\end{tabular}

G47 $\Delta$ is another modified herpes OV with a deletion in the $\gamma 34.5$ gene encoding ICP34.5 protein and with the insertion of the Escherichia coli LacZ gene inactivating the ICP6 gene. G47 $\triangle$ demonstrated a greater replication capability and a higher antitumor efficacy than G207. G47 $\Delta$ exhibited a significant efficacy against ATC xenografts [68].

In Table 1 are summarized the studies performed with OVs in anaplastic or poorly differentiated thyroid carcinoma models.

\section{Mechanisms of action of OVs}

Activation of cell death pathways by OVs \& immune response

The mechanisms of action of OVs are not completely understood. A direct lytic effect has been theorized in the initial studies. More recent studies have proved indirect effects of OVs. These include the induction of innate and adaptive immune response against cancer cells and the re-shaping of tumor microenvironment (TME), (Figure 1) such as by inhibition of tumor angiogenesis [70,71]. All these mechanisms effectively contribute to the antitumoral effects [1].

The neoplastic cells in response to the infection of OVs activate different cell death pathways depending on the virus type, the cell type or a combination of both [72]. Cell death pathways include apoptosis, pyroptosis (caspase-1-dependent cell death), autophagic cell death, immunogenic cell death (ICD) and necrosis. The cell death pathway(s) activated by the infection with OVs, excluding apoptosis, are highly immunogenic [73,77].

ICD is characterized by the release of cytokines, tumor-associated antigens and other danger signals, including damage-associated molecular pattern molecules and pathogen-associated molecular pattern molecules, stimulating an immune response against infected and noninfected cancer cells [73,77]. Dying cells release a variety of cytokines into the local environment, such as IFNs, TNF- $\alpha$ and interleukins that further promote immune response [78-80].

Due to the cell-type dependent effects of the OVs on the activation of cell death pathways it is necessary to assess the effects of a specific virus in the context of the neoplastic cell type. Several studies have evaluated the effect of virotherapy in ATC cells. In particular, dl922-947 induced the activation of a programmed cell death that shares some features with apoptosis [56-58]. However, the treatment with caspase inhibitor zVAD-fmk was not able to modify cell survival, suggesting the involvement of a non-apoptotic pathways in $d l 922-947$-induced death of ATC cells [58]. Authors have also excluded the activation of autophagy or the death by mitotic catastrophe [57]. The activation of a PARP-mediated cell death (parthanatos) in ATC cells infected with dl922-947 has been also 


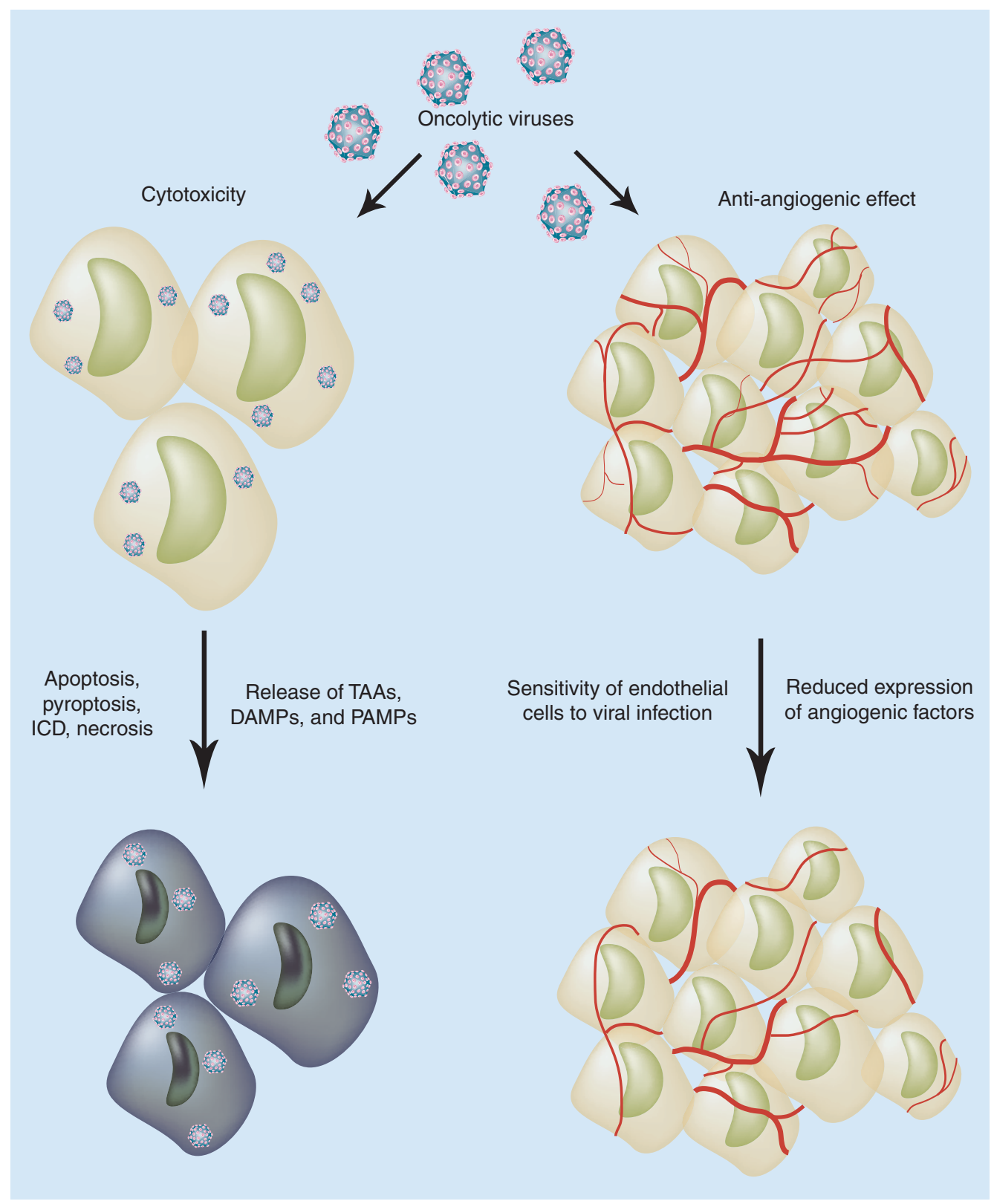

Figure 1. Effects of oncolytic viruses on tumor microenvironment in ATC.

analysed. AIF is a key mediator of parthanatos: upon PARP-1 activation, AIF translocates from the mitochondria to the nucleus. ATC cells infected with $d l 922-947$ did not show any AIF translocation, leading to the exclusion of parthanatos activation [58].

So far, the involvement of pyroptosis or ICDs has not been evaluated in ATC cells infected with dl922-947, although the assessment of the cell death pathway activated by dl922-947 in ATC cells would be helpful to develop rationally-based combinatorial treatments.

The involvement of immune response in the antitumoral activity of OVs is now accepted. However, most of the preclinical studies on OVs have been performed using immunodeficient mice and human xenograft. The use of these models limits the study of the immune response, especially the adaptive response. Moreover, the lack of immune competent animal experimental models (lack of availability of mouse syngeneic cancer cells to engraft), or the natural tropism of the virus for human cells, but not mouse cells, represent a hurdle to these studies. 
Nevertheless, experiments to address the immune response in ATC experimental tumors have been performed. In ATC xenografts (lacking the T cell-mediated immunity), dl922-947 adenovirus reduces the production of the monocyte attracting chemokine CCL2 and tumor-associated macrophage (TAM) density in vivo. TAM depletion in $\mathrm{d}$ l922-947-treated tumors is associated with increased expression of Nos2, a marker of pro-inflammatory M1 macrophages [81]. Moreover, an increased expression of Ifng mRNA encoding the protein IFN $\gamma$ was observed. IFN $\gamma$ may induce a switch toward a pro-inflammatory macrophage M1 phenotype [64]. However, this model is inadequate to fully recapitulate the mediated anti-tumor response elicited by virotherapy. A confirmation in an alternative immune competent model is probably required, although the preclinical studies performed with this virus are already sufficient for a clinical trial that could contribute to clarify this issue.

\section{Modulation of TME by OVs}

An effect on TME has been described for several OVs, including VACVs, parvovirus and adenoviruses in preclinical models and in clinical studies [81-83]. It involves the targeting of tumor-associated endothelial cells leading to a rapid destruction of the tumor vasculature and loss of perfusion within the tumor. The mechanisms underlying this process are poorly understood. Studies have shown that despite apparent profound and rapid loss of tumor perfusion, complete responses remain elusive and tumor regrowth occurs, suggesting a revascularization and neo-angiogenesis of treated tumors $[82,83]$.

Oncolytic vaccinia virus possesses an anti-angiogenic potential and this effect can be exploited for synergistic combination with different antitumoral agents [83].

Proliferating human umbilical vein endothelial cells (HUVEC) and immortalized Kaposi sarcoma-derived human endothelial cells (KS-IMM) are sensitive to OV H-1PV. Although the cells undergo an abortive infection which does not lead to viral progeny production and spread, the infection of highly angiogenic KS-IMM cells significantly reduces tumor-forming ability after sub-cutaneous implantation and the infection inhibits the expression of the key angiogenesis factor, VEGF [74].

A significant reduction in microvessel density and a decrease in CD31 mRNA (marker of endothelial cells) expression was observed in ATC xenograft tumors, confirming an innate anti-angiogenic effect of dl922-947 [58]. Another study not only confirmed this effect in ATC xenografts but further showed that dl922-947 treatment of ATC cells impairs the secretion of the pro-angiogenic chemokine IL-8 [81]. These findings were substantiated by in vivo results, showing that $d l 922-947$ treatment of tumor xenografts established in athymic mice reduces $I L 8$ and Cd31 mRNA level and overall the tumor microvascular density.

\section{Combination therapies with OVs}

OVs \& chemotherapeutic drugs

Despite the efficacy of OVs against cancer cells, it is unlikely that tumor eradication could be obtained using OVs as single agents. In the initial studies, the combination of OVs with standard chemotherapeutics has been tested as a strategy to increase their efficacy [75]. The development of novel and more effective OVs, together with a better understanding of the interactions between OVs and tumor cells, has led to combinatorial treatments based on rational approaches [84].

The oncolytic adenovirus ONYX-015 has been tested in association with doxorubicin and paclitaxel against ATC cells. The combined treatment activated cell death more efficiently with respect to single treatments, likely because $E 1 A$ gene products act on cell death pathways activation [11]. Lovastatin, a cholesterol-lowering drug also acting as an inhibitor of $\mathrm{p} 21 /$ ras activity, was used in conjunction with $d l 1520$ to improve viral cytotoxicity. This combination increased the death of ATC cells and viral replication [52].

An obstacle to an efficient infection of tumor cells by OVs is represented by the abnormal tumor vascular structure and by the increased interstitial tumor pressure [1]. To improve viral distribution and diffusion, dl922-947 has been used in combination with bevacizumab, a humanized anti-VEGF antibody. The treatment of ATC xenografts with bevacizumab, prior to virus administration, normalized the vascular structure, therefore reducing interstitial pressure and allowing for a more efficient distribution of the virus within the tumor [55].

A typical feature of ATC cells infected by d $1922-947$ is represented by the increase of DNA content $(>4 N)$ and the accumulation of the cells in the G2/M phase of the cell cycle [77]. This effect is probably due to the abrogation of multiple cell cycle checkpoints by viral proteins. Indeed, $E 1 B$ gene products prevent cell death and in combination with E4-gene products block the activation of the cell cycle checkpoints. Libertini $e t$ al. hypothesised that the block of the mitotic checkpoints could enhance the efficacy of dl922-947 [58]. The block of mitotic kinase Aurora B, 
that is overexpressed in ATC, reduces the tumor growth [85]. A specific Aurora B kinase inhibitor (AZD1152) has been used in combination with $d l 922-947$ showing additive/synergistic killing effects and a significant inhibition of tumor growth [57].

DNA damage response (DDR) represents a cellular defence against viruses. To hinder viral infection, the viral DNA is processed by the host cell as a damaged DNA. Viruses have evolved strategies to counteract DDR activity, which include degradation or mislocalization of key players involved in DDR [86-88]. In ATC cells, dl922-947 triggers an inefficient DDR, with an accumulation of $\gamma \mathrm{H} 2 \mathrm{AX}$, a marker of DNA damage [71]. A specific-inhibitor of ATM (KU55933) increased the replication and the oncolytic activity of dl922-947, confirming that the DDR acts as a cellular defense and its inhibition could enhance the effects of OVs [56].

Other DNA repair pathways protect the integrity of the genome. Poly (ADP-ribose) polymerase 1 and 2 (PARP-1 and PARP-2) are key mediators in base excision repair (BER) pathway. PARP-1 is also involved in other DNA damage repair processes, including the repair of DNA double strand breaks [89]. During viral infections, cells are pushed toward S phase inducing the accumulation of single strand breaks (SSBs) as unscheduled DNA synthesisassociated DNA lesions [90]. Therefore, Passaro et al. have hypothesized that dl922-947 infection could induce PARP-1 activation. A robust protein PARylation in ATC cells infected with dl922-947 was observed, confirming their hypothesis. Cells defective in homologous recombination are highly sensitive to PARP-1 inhibition [91], due to an increasing genomic instability and cell death (synthetic lethality). Passaro et al. further demonstrated that dl922-947 impaired DDR and the combined treatment of $d l 922-947$ with a specific PARP inhibitor (Olaparib) was effective against ATC cells. Indeed, this treatment, mimicking synthetic lethality, greatly increased DNA damage accumulation and cell death in ATC cells and tumor xenografts [58].

Ionizing radiation (IR) induces DNA damage and a subsequent block of the cell cycle with accumulation of cells either in G1 or G2 phase of the cell cycle, depending on the integrity of cell cycle checkpoints. Due to capability of oncolytic adenoviruses to greatly interfere with DDR, the association with IR has been tested in ATC models.

In an early study, the combination of ONYX-015 and radiotherapy was tested showing an increased cell killing and a delayed growth of ATC xenograft [51]. However, this initial study was only descriptive and the mechanisms leading to the enhanced cell killing were not investigated. In another study, Passaro et al. have shown that the IR combination enhances the effect of $d l 922-947$ against ATC cells in vitro. In particular, a synergistic cell killing was observed only when irradiation was administered prior to viral infection and the efficacy of the combined treatment was confirmed in ATC xenografts [56].

The preclinical evaluation of OVs in combination with other agents for ATC treatment has been performed with oncolytic HSVs.

Lin and colleagues have tested oncolytic herpes viruses G207 and NV1023 in combination with adriamycin or paclitaxel in ATC cell lines [92]. The combination of G207 with paclitaxel showed synergistic effects in both cell lines. Although G207 viral entry and replication were not enhanced by paclitaxel, G207 in combination with paclitaxel significantly increased microtubule acetylation, mitotic arrest, aberrant chromatid separation, inhibition of cell cycle progression and apoptosis. The treatment of athymic mice bearing ATC xenografts confirmed the efficacy of this approach [92]. After the publication of this study it has been discovered that the ATC cell lines (KAT4 and DRO90-1) used by the authors to assess the efficacy of G207 in combination with paclitaxel, do not originate from thyroid carcinoma, and therefore it would be useful to confirm in cells of proved thyroid origin [93].

In Table 1 are summarized the combination studies performed with OVs.

Although the data obtained using OVs in combination with antineoplastic agents are encouraging, it is not possible to exclude that other OVs might display a superior cell killing in combinatorial treatments. Moreover, additional data might be useful for future clinical studies, such as the use of orthotopic models of ATC and in vivo imaging experiments to gain more data on viral administration, viral diffusion and spread to normal tissues.

Overall, these studies have confirmed that combinatorial approaches hold a potential for the treatment of ATC, suggesting a clinical evaluation. Currently used chemotherapeutic agents, such as doxorubicin and paclitaxel, have been tested in combination only with ONYX-015 and G207 and the results obtained in these studies need a confirmation with more potent OVs [11].

Other specific inhibitors have not been tested or are not used for the treatment of solid tumors [56-58], making the clinical evaluation of these combinations less likely.

PARP inhibitors are already used for the treatment of ovarian cancer patients with BRCA2- gene mutations and a clinical evaluation of the combined treatment with $d\lfloor 922-947$ could be envisaged [58]. Finally, multimodality treatment of ATC already includes IR, therefore clinical evaluation of this rationally based combination is feasible [58]. 


\section{Future perspective}

Cancer immunotherapy is a clinically validated treatment for many solid tumors. Immunotherapy includes cancer vaccines, adoptive transfer of activated $\mathrm{T}$ or NK cells, the use of CAR T cells, OVs and antibodies blocking the so called immune checkpoint inhibitors, such as anti-CTLA4 and anti-PD-1/PD-L1 antibodies [94,95].

Immune checkpoint molecules are used by the immune system to maintain homeostasis, for instance, for the prevention of pathologic autoimmunity. Conversely, in tumors these signals are upregulated, allowing tumors to evade protective immune responses [95]. Immune checkpoint antagonists have been approved for the clinical use against different lesions [96]. However, it is accepted that this approach works better in neoplastic lesions with an existing antitumor immune response, whereas tumors with a low expression of PD-1/PDL-1 and minimal immune cells infiltration are less responsive. Moreover, even in neoplastic lesions such as melanoma, where checkpoint inhibitors have proved to be effective as single agents, only $20-25 \%$ of patients exhibit a durable response [96]. However, up to $50 \%$ tumor regressions have been observed using anti CTLA- 4 in combination with anti PD1 [97], indicating that immune checkpoint inhibitors should not be used as single agents and suggesting their use in combination with other agents capable to stimulate a robust immune response against neoplastic cells.

Taking into account the capability of OVs to induce an immunogenic cell death and the subsequent activation of innate and/or adaptive immune response [77,78], OVs and immune checkpoint blockade could synergise in stimulating the immune system against the tumor [98]. Indeed, OVs treatment could stimulate the recruitment of effector T cells into the TME, whereas immune checkpoint blockade may sustain the potency of tumor infiltrating lymphocytes, TAM with an inflammatory M1 phenotype and NKs via the removal of inhibitory signals [95].

The efficacy of this strategy has been confirmed in several studies in different types of tumors [99].

ATC tissue is composed of about $60 \%$ of TAMs mixed with cancer cells and the increased number of TAMs in poorly differentiated carcinomas is associated with a decreased survival [100], probably reflecting an immunosuppression and trophic activity (M2 type) in response to Th2 cytokines (i.e., IL-10, TGF- $\beta$ ) [64]. Overall, in aggressive thyroid tumors a protumoral role of the infiltrating immune cells has been already established. Moreover, ATC cells express high levels of the enzymeindoleamine 2,3-dioxygenase (IDO1) that catalyzes the conversion of the amino acid tryptophan to the immunosuppressive molecule kynureine, thus causing halted growth of $\mathrm{T}$ cells [101].

PD-L1 expression is a potential biomarker to predict the response to anti-PD-1 or anti-PD-L1 agents [102]. In thyroid carcinomas a significant increase in membrane PD-L1 positivity was correlated with high risk of aggressive disease, distant metastasis or death. The combined cytoplasmic and membrane PD-L1 positivity was also associated with poor prognosis [103]. In another study PD-L1 has been shown to be highly expressed in a subset of patients with ATC [104].

Overall, these findings suggest a role for immunotherapeutic agents in patients with refractory thyroid cancer [105].

A potential immunostimulatory activity of $d l 922-947$ has been shown in ATC models, thus making this virus an attractive candidate for combination treatments with anti-CTLA- 4 and anti-PD-1/PD-L1 antibodies.

It is worth to note that the efficacy of $d l 922-947$ has been assessed in immunocompromised animal models, obtaining only data suggestive of an NK cell activation [81]. Indeed, the use of immunocompromised models precludes to study the role of the immune adaptive response in adenoviral virotherapy, therefore data obtained in immune compromised models need to be confirmed in immune competent animals.

The studies in an immune competent setting require the availability of the specific murine cancer cell lines. Unfortunately, regarding the ATC only a limited number of cell lines are available [106,107]. Moreover, the use of murine cells encounters another problem when using viruses with human tropism. In fact, human-tropism viruses (i.e., adenoviruses, HSVs, etc) poorly replicate in mouse cell line, leading to an underestimation of the oncolytic effect of the virus. The use of more sophisticated immune competent models such as the humanized model is desirable. In humanized setting, the mice can be engrafted both with human cancer cells and human immune cells. However, this system is both challenging and expensive.

Alternatively, in vitro experiments may allow to investigate the effects of dl922-947 and immune checkpoint inhibitors on the stimulation of human NK cells, monocyte and monocyte-derived macrophages. Although these studies do not entirely recapitulate the complex interaction of the tumor microenvironment, data could be sufficient to understand the potential therapeutic efficacy of the combination. 


\section{Conclusion}

The lack of effective therapies for ATC emphasizes the need for novel treatments and the use of OVs was hypothesized since initial studies with ONYX-015 [11,51,52], and OVs could be helpful in improving disease control and survival of ATC patients.

Several issues remain to be addressed, such as which OV will be the more effective for ATC treatment and/or which administration route (intratumoral or I.V.) should be used. The effects of the OV-based treatments on distant metastases and the efficacy of OVs in combination also require assessement.

Clinical trials could be helpful to address the open questions, however, ATC is a rare lesion, found in only $2 \%$ of thyroid tumors, and most of the patients have a short survival time, making a clinical study difficult [10]. A compassionate treatment of ATC patients with $d l 922-947$ could help in gaining clinical and efficacy data.

Although no clinical trials based on OVs for the treatment of ATC are in progress, viruses have already been used in patients with thyroid carcinoma. Indeed, in clinical trials aiming for the evaluation of OVs against solid tumors, thyroid carcinoma patients have been treated. These trials were not designed for the treatment of ATC and the virus was not previously tested in ATC models. In a Phase II clinical trial investigating, in patients with advanced cancers, the effects of intratumoral administration of a reovirus (REOLYSIN ${ }^{\circledR}$ ) in combination with low-dose radiation, a single patient with thyroid carcinoma was enrolled and a partial response was observed [76].

Viruses with different mechanisms of action with respect of OVs have been also tested in clinical trial for thyroid carcinoma. VB-111 is a nonreplicating adenovirus [108], expressing a Fas-chimera transgene under the control of murine preproendothelin promoter that is active only against tumor vasculature. VB-111 is currently under evaluation in a Phase II clinical trials (ClinicalTrials. gov Identifier: NCT01229865, VB-111) in patients with advanced differentiated thyroid cancer. In this study, 44\% of patients experienced tumor regression. Despite the lack of replication of VB-111 the results of this study could be useful for the future clinical use of replicating OVs, although the different mechanism of action of VB-111 does not allow to obtain conclusive indication on the therapeutic potential of OVs in ATC patients. Overall, these data obtained treating thyroid carcinoma patients with viruses are important for future studies.

Several OVs have been evaluated in preclinical studies, among these the adenoviral mutant $d l 922-947$ is the most studied in ATC models, and its efficacy has been demonstrated in combination with different agents, including IR, making this virus a promising candidate for clinical studies [56-58,81].

In conclusion, the concept of OV-based cancer therapy is now shifted from the selective killing of cancer cells to immunostimulatory agents, opening a novel operational scenario and to an efficient coupling with immune checkpoint inhibitors. The efficacy of OVs has been clearly confirmed in the ATC preclinical scenario, strongly supporting its clinical use for the treatment of ATC.

\section{Executive summary}

- Oncolytic viruses (OVs) selectively infect and lyse cancer cells.

- Three OVs are used for cancer treatment in the world and other OVs are currently under investigation in clinical studies.

- T-VEC (talimogene laherparepvec) has been approved in Europe and the USA.

- OVs can be considered as immunostimulatory agents and their use in combination with inhibitors of immune checkpoints is under evaluation with promising results.

- Anaplastic thyroid carcinomas are resistant to currently available therapies and require more effective treatments.

- Preclinical studies have demonstrated the potential benefits of OVs against ATC in vitro and in vivo, both as single agents or in combinatorial treatments.

Financial \& competing interests disclosure

The authors have no relevant affiliations or financial involvement with any organization or entity with a financial interest in or financial conflict with the subject matter or materials discussed in the manuscript. This includes employment, consultancies, honoraria, stock ownership or options, expert testimony, grants or patents received or pending, or royalties.

No writing assistance was utilized in the production of this manuscript. 


\section{Open access}

This work is licensed under the Creative Commons Attribution 4.0 License. To view a copy of this license, visit http://creativecommons.org/licenses/by/4.0/

\section{References}

Papers of special note have been highlighted as: • of interest; $\bullet \bullet$ of considerable interest

1. Russell SJ, Peng KW, Bell JC. Oncolytic virotherapy. Nat. Biotechnol. 30(7), 658-670 (2012).

2. Howells A, Marelli G, Lemoine NR et al. Oncolytic viruses-interaction of virus and tumor cells in the battle to eliminate cancer. Front. Oncol. 7, 195 (2017).

3. Vacchelli E, Eggermon A, Sautes-Fridman C et al. Trial watch: oncolytic viruses for cancer therapy. Oncoimmunology 2(6), e24612 (2013).

4. Babiker HM, Riaz IB, Husnain M et al. Oncolytic virotherapy including Rigvir and standard therapies in malignant melanoma. Oncolytic Virother. 6, 11-18 (2017).

5. Garber K. China approves world's first oncolytic virus therapy for cancer treatment. J. Natl Cancer Inst. 98(5), 298-300 (2006).

6. Andtback RH, Kaufman HL, Collichi F et al. Talimogene laherparepvec improves durable response rate in patients with advanced melanoma. J. Clin. Oncol. 33(825), 2780-2788 (2015).

-• Reports the data obtained in a Phase III clinical trial showing that talimogene laherparepvec was the first oncolytic immunotherapy with a clear therapeutic benefit against melanoma, particularly in advanced stages.

7. Taccaliti A, Silvetti F, Palmonella G, Boscaro M. Anaplastic thyroid carcinoma. Front. Endocrinol. (Lausanne) 5(3), 84 (2012).

8. Hundahl SA, Fleming ID, Fremgen AM, Menck HR. A National Cancer Database report on 53,856 cases of thyroid carcinoma treated in the U.S., 1985-1995, Cancer 83(12), 2638-2648 (1998).

9. Kitamura Y, Shimizu K, Nagahama M et al. Immediate causes of death in thyroid carcinoma: clinico pathological analysis of 161 fatal cases. J. Clin. Endocrinol. Metab. 84(11), 4043-4049 (1999).

10. Smallridge RC, Copland JA. Anaplastic thyroid carcinoma: pathogenesis and emerging therapies. Clin. Oncol. (R. Coll. Radiol.) 22(6), 486-497 (2010).

11. Portella G, Scala S, Vitagliano D et al. ONYX-015, an E1B gene-defective adenovirus, induces cell death in human anaplastic thyroid carcinoma cell lines. J. Clin. Endocrinol. Metab. 87(6), 2525-2531 (2002).

12. Dock, G. The influence of complicating diseases on leukemia. Am. J. Med. Sci. 127, 563-592 (1904).

13. De Pace N. Sulla scomparsa di un enorme cancro vegetante del collo dell'utero senza cura chirurgica. Ginecologia 9, 82-89 (1921).

14. Bierman HR, Crile DM, Dod KS et al. Remissions in leukemia of childhood following acute infectious disease: Staphylococcus and Streptococcus, varicella, and feline panleukopenia. Cancer 6(3), 591-605 (1953).

15. Anderson WF, Blaese RM, Culver K. The ADA human gene therapy clinical protocol: points to consider response with clinical protocol. Hum. Gene Ther. 1(3), 331-362 (1990).

16. Martuza RL, Malick A, Markert JM, Ruffner KL, Coen DM. Experimental therapy of human glioma by means of a genetically engineered virus mutant. Science 252(5007), 854-856 (1991)

-. This landmark study reports the data obtained with a genetically modified Herpes Simplex Virus-1 (TK-mutated and with reduced neurotoxicity). The virus was shown to replicate selectively in cancer cells.

17. Bischoff JR, Kirn DH, Williams A et al. An adenovirus mutant that replicates selectively in p53-deficient human tumor cells. Science 274(2586), 373-376 (1996).

-. In this study a E1B gene-mutated adenovirus was described. The data have opened the possibility that mutant adenoviruses can be used for cancer treatment.

18. O'Shea CC, Johnson L, Bagus B et al. Late viral RNA export, rather than $\mathrm{p} 53$ inactivation, determines ONYX-015 tumor selectivity. Cancer Cell 6(6), 611-623 (2004).

- Demonstrates that loss of E1B-55K-mediated late viral RNA export, restricting the replication of d11520 to tumor cells that provide the RNA export function of E1B-55K.

19. Liu TC, Galanis E, Kirn D. Clinical trial results with oncolytic virotherapy: a century of promise, a decade of progress. Nat. Clin. Pract. Oncol. 4(2), 101-117 (2007).

20. Larson C, Oronsky B, Scicinski J et al. Going viral: a review of replication-selective oncolytic adenoviruses. Oncotarget 6(24), 19976-19989 (2015).

21. Guo ZS, Liu Z, Kowalsky S et al. Oncolytic immunotherapy: conceptual evolution, current strategies, and future perspectives. Front. Immunol. 8, 555 (2017).

22. Topalian SL, Drake CG, Pardoll DM. Immune checkpoint blockade: a common denominator approach to cancer therapy. Cancer Cell. 27(4), 450-461 (2015). 
23. Raftery N, Stevenson NJ. Advances in anti-viral immune defence: revealing the importance of the IFN JAK/STAT pathway. Cell. Mol. Life Sci. 74(14), 2525-2535 (2017).

24. Kaufman HL, Kohlhapp FJ, Zloza A. Oncolytic viruses: a new class of immunotherapy drugs. Nat. Rev. Drug Discov. 14(9), 642-662 (2015).

25. Nguyen TL, Abdelbary $\mathrm{H}$, Arguello $\mathrm{M}$ et al. Chemical targeting of the innate antiviral response by histone deacetylase inhibitors renders refractory cancers sensitive to viral oncolysis. Proc. Natl Acad. Sci. USA 105(39), 14981-14986 (2008).

26. Wang G, Barrett JW, Stanford M et al. Infection of human cancer cells with myxoma virus requires akt activation via interaction with a viral ankyrin-repeat host range factor. Proc. Natl Acad. Sci. USA 103(12), 4640-4645 (2006).

27. Mundi N, Um S, Yoo J et al. The control of anaplastic thyroid carcinoma cell lines by oncolytic poxviruses. Virus Res. 190, 53-59 (2014).

28. Haddad D. Genetically engineered Vaccinia viruses as agents for cancer treatment, imaging, and transgene delivery. Front. Oncol. 23(7), 96 (2017).

29. Buller RM, Smith GL, Cremer K et al. Decreased virulence of recombinant vaccinia virus expression vectors is associated with a thymidine kinase-negative phenotype. Nature 317(6040), 813-815 (1985).

30. Guse K, Cerullo V, Hemminki A. Oncolytic vaccinia virus for the treatment of cancer. Expert Opin. Biol. Ther. 11(5), $595-608$ (2011).

31. Thorne SH, Hwang TH, O'Gorman WE et al. Rational strain selection and engineering creates a broad-spectrum, systemically effective oncolytic poxvirus, JX-963. J. Clin. Invest. 117(11), 3350-3358 (2007).

-. Describes the design and evaluation of JX-963. The virus was engineered to target cancer cells with activation of the transcription factor E2F and the EGFR pathway by deletion of the thymidine kinase and vaccinia growth factor genes.

32. Zhang Q, Yu YA, Wang E et al. Eradication of solid human breast tumors in nude mice with an intravenously injected light-emitting oncolytic vaccinia virus. Cancer Res. 67(20), 10038-10046 (2007).

33. Lin SF, Yu Z, Riedl C, Woo Y et al. Treatment of anaplastic thyroid carcinoma in vitro with a mutant vaccinia virus. Surgery 142(6), 976-983 (2007).

34. Lin SF, Price DL, Chen CH et al. Oncolytic vaccinia virotherapy of anaplastic thyroid cancer in vivo. J. Clin. Endocrinol. Metab. 93(11), 4403-4407 (2008).

35. Gholami S, Haddad D, Chen CH et al. Novel therapy for anaplastic thyroid carcinoma cells using an oncolytic vaccinia virus carrying the human sodium iodide symporter. Surgery 150(6), 1040-1047 (2011).

36. Conrad SJ, El-Aswad M, Kurban E et al. Oncolytic tanapoxvirus expressing FliC causes regression of human colorectal cancer xenografts in nude mice. J. Exp. Clin. Cancer Res. 34, 19, (2015).

37. Russell SJ, Peng KW. Measles virus for cancer therapy. Curr. Top Microbiol. Immunol. 330, 213-241 (2009).

38. Ammayappan A, Russell SJ, Federspiel MJ. Recombinant mumps virus as a cancer therapeutic agent. Mol. Ther. Oncolytics 10(3), 16019 (2016).

39. Schirrmacher V. Fifty years of clinical application of Newcastle Disease virus: time to celebrate! Biomedicines 4(3), pii: E16 (2016).

40. Rota JS, Wang ZD, Rota PA, Bellini WJ. Comparison of sequences of the H, F, and N coding genes of measles virus vaccine strains. Virus Res. 31(3), 317-330 (1994).

41. Yamakawa M, Yamada K, Tsuge T et al. Protection of thyroid cancer cells by complement-regulatory factors. Cancer 73(11), 2808-2817 (1994).

42. Anderson BD, Nakamura T, Russell SJ, Peng KW. High CD46 receptor density determines preferential killing of tumor cells by oncolytic measles virus. Cancer Res. 64(14), 4919-4926 (2004).

43. Reddi HV, Madde P, McDonough SJ et al. Preclinical efficacy of the oncolytic measles virus expressing the sodium iodide symporter in iodine non-avid anaplastic thyroid cancer: a novel therapeutic agent allowing non invasive imaging and radioiodine therapy. Cancer Gene Ther. 19(9), 659-665 (2012).

44. Nüesch JP, Lacroix J, Marchini A, Rommelaere J. Molecular pathways: rodent parvoviruses mechanisms of oncolysis and prospects for clinical cancer treatment. Clin. Cancer Res. 18(13) 3516-3523 (2012).

45. Angelova AL, Witzens-Harig M, Galabov AS, Rommelaere J. The oncolytic virotherapy era in cancer management: prospects of applying H-1 Parvovirus to treat blood and solid cancers. Front. Oncol. 7, 93 (2017).

46. Fusco A, Berlingieri MT, Di Fiore PP et al. One- and two-step transformations of rat thyroid epithelial cells by retroviral oncogenes. Mol. Cell. Biol. 7(9), 3365-3370 (1987).

47. Portella G, Ferulano G, Santoro M et al. The Kirsten murine sarcoma virus induces rat thyroid carcinomas in vivo. Oncogene 4(2), 181-188 (1989).

48. Santoro M, Melillo RM, Grieco M, Berlingieri MT, Vecchio G, Fusco A. The TRK and RET tyrosine kinase oncogenes cooperate with ras in the neoplastic transformation of a rat thyroid epithelial cell line. Cell Growth Differ. 4(2), 77-84 (1993).

49. Maroun J, Muñoz-Alía M, Ammayappan A, Schulze A, Peng KW, Russell S. Designing and building oncolytic viruses. Future Virol. 12(4), 193-213 (2017). 
50. Guo ZS, Thorne SH, Bartlett DL. Oncolytic virotherapy: molecular targets in tumor-selective replication and carrier cell-mediated delivery of oncolytic viruses. Biochim. Biophys. Acta. 1785(2), 217-231 (2008).

51. Portella G, Pacelli R, Libertini S et al. ONYX-015 enhances radiation-induced death of human anaplastic thyroid carcinoma cells. J. Clin. Endocrinol. Metab. 88(10), 5027-5032 (2003).

52. Libertini S, Iacuzzo I, Ferraro A et al. Lovastatin enhances the replication of the oncolytic adenovirus d1 1520 and its antineoplastic activity against anaplastic thyroid carcinoma cells. Endocrinology 148(11), 5186-5194 (2007).

53. Heise $\mathrm{C}$, Hermiston $\mathrm{T}$, Johnson $\mathrm{L}$ et al. An adenovirus E1A mutant that demonstrates potent and selective systemic anti-tumoral efficacy. Nat. Med. 6(10), 1134-1139 (2000).

- Described the E1A mutant oncolytic adenovirus d1922-947. This second generation mutant demonstrated a superior in vivo potency relative to other adenoviruses.

54. Sherr CJ, McCormick F. The RB and p53 pathways in cancer. Cancer Cell 2(2), 103-112 (2002).

55. Libertini S, Iacuzzo I, Perruolo G et al. Bevacizumab increases viral distribution in human anaplastic thyroid carcinoma xenografts and enhances the effects of E1A-defective adenovirus dl922-947. Clin. Cancer Res. 14(20), 6505-6514 (2008).

56. Passaro C, Abagnale A, Libertini $S$ et al. Ionizing radiation enhances dl $922-947$-mediated cell death of anaplastic thyroid carcinoma cells. Endocr. Relat. Cancer 20(5), 633-647 (2013).

57. Libertini S, Abagnale A, Passaro C et al. AZD1 152 negatively affects the growth of anaplastic thyroid carcinoma cells and enhances the effects of oncolytic virus dl922-947. Endocr. Relat. Cancer 18(1), 129-141 (2011).

58. Passaro C, Volpe M, Botta G et al. PARP inhibitor olaparib increases the oncolytic activity of dl922-947 in in vitro and in vivo model of anaplastic thyroid carcinoma. Mol. Oncol. 9(1), 78-92 (2014).

59. Abbosh PH, Li X, Li L et al. A conditionally replicative, Wnt/beta-catenin pathway-based adenovirus therapy for anaplastic thyroid cancer. Cancer Gene Ther. 14(4), 399-408 (2007).

60. Hibma MH, Real NC, Wiles A et al. Increased apoptosis and reduced replication efficiency of the E3 region-modified dl309 adenovirus in cancer cells. Virus Res. 145(1), 112-120 (2009).

61. Advani SM, Chung Y, Yan GY et al. Replication-competent, non neuroinvasive genetically engineered herpes virus is highly effective in the treatment of therapy resistant experimental human tumors. Cancer Res. 59(9), 2055-2058 (1999).

62. Todo T. Oncolytic virus therapy using genetically engineered herpes simplex viruses. Front. Biosci. 13, 2060-2064 (2008).

63. Varghese S, Rabkin SD. Oncolytic herpes simplex virus vectors for cancer virotherapy. Cancer Gene Ther. 9(12), 967-978 (2002).

64. Sica A, Mantovani A. Macrophage plasticity and polarization: in vivo veritas. J. Clin. Invest. 122(3), 787-795 (2012).

65. Goldstein DJ, Weller SK. Factor(s).present in herpes simplex virus type1-infected cells can compensate for the loss of the large subunit of the viral ribonucleotide reductase: characterization of an ICP6 deletion mutant. Virology 166(1), 41-51 (1988).

66. Lin SF, Gao SP, Price DL et al. Synergy of a herpes oncolytic virus and paclitaxel for anaplastic thyroid cancer. Clin. Cancer Res. 14(5), 1519-1528 (2008).

67. Yu Z, Eisenberg DP, Singh B, Shah JP, Fong Y, Wong RJ. Treatment of aggressive thyroid cancer with an oncolytic herpes virus. Int. J. Cancer 112(3), 525-532 (2004).

68. Wang JN, Xu LH, Zeng WG, Hu P, Rabkin SD, Liu RR. Treatment of human thyroid carcinoma cells with the g47delta oncolytic herpes simplex virus. Asian Pac. J. Cancer Prev. 16(3), 1241-1245 (2015).

69. Reddi HV, Madde P, Reichert-Eberhardt AJ et al. ONYX-411, a conditionally replicative oncolytic adenovirus, induces cell death in anaplastic thyroid carcinoma cell lines and suppresses the growth of xenograft tumors in nude mice. Cancer Gene Ther. 15(11), 750-757 (2008).

70. Breitbach CJ, Paterson JM, Lemay CG et al. Targeted inflammation during oncolytic virus therapy severely compromises tumor blood flow. Mol. Ther. 15(9), 1686-1693 (2007).

71. Liu TC, Hwang T, Park BH, Bell J, Kirn DH. The targeted oncolytic poxvirus jx-594 demonstrates antitumoral, antivascular, and anti-hbv activities in patients with hepatocellular carcinoma. Mol. Ther. 16(9), 1637-1642 (2008).

72. Bartlett DL, Liu Z, Sathaiah M et al. Oncolytic viruses as therapeutic cancer vaccines. Mol. Cancer 12(1), 103 (2013).

73. Inoue H, Tani K. Multimodal immunogenic cancer cell death as a consequence of anticancer cytotoxic treatments. Cell Death Differ. 21(1), 39-49 (2014).

74. Lavie M, Struyf S, Stroh-Dege A et al. Capacity of wild-type and chemokine-armed parvovirus H-1PV for inhibiting neo-angiogenesis. Virology 447(1-2), 221-232 (2013).

75. Bressy C, Benihoud K. Association of oncolytic adenoviruses with chemotherapies: an overview and future directions. Biochem. Pharmacol. 90(2), 97-106 (2014).

76. Guan M, Romano G, Coroniti R, Henderson EE. Progress in oncolytic virotherapy for the treatment of thyroid malignant neoplasm. J. Exp. Clin. Cancer Res. 33, 91 (2014). 
77. Guo ZS, Liu Z, Bartlett DL. Oncolytic immunotherapy: dying the right way is a key to eliciting potent antitumor immunity. Front. Oncol. 4, 74 (2014).

78. Benencia F, Courreges MC, Conejo-Garcia JR et al. HSV oncolytic therapy upregulates interferon-inducible chemokines and recruits immune effector cells in ovarian cancer. Mol. Ther. 12(6), 789-802 (2005).

79. Donnelly O G, Errington-Mais F, Steele L et al. Measles virus causes immunogenic cell death in human melanoma. Gene Ther. 20(1), 7-15 (2013).

80. Wongthida P, Diaz R M, Galivo F et al. Type III IFN interleukin-28 mediates the antitumor efficacy of oncolytic virus VSV in immune-competent mouse models of cancer. Cancer Res. 70(11), 4539-4549 (2010).

81. Passaro C, Borriello F, Vastolo V et al. The oncolytic virus $d l 922-947$ reduces IL-8/CXCL8 and MCP-1/CCL2 expression and impairs angiogenesis and macrophage infiltration in anaplastic thyroid carcinoma. Oncotarget 7(2), 1500-1515 (2016).

82. Breitbach CJ, Arulanandam R, De Silva N et al. Oncolytic vaccinia virus disrupts tumor-associated vasculature in humans. Cancer Res. 73(4), 1265-1275 (2013).

- In this study the antiangiogenic activity of oncolytic vaccinia virus JX-594 was demonstrated showing that the virus triggers acute tumor vascular disruption and decreased perfusion in patients with hypervascular liver tumors.

83. Breitbach CJ, Paterson JM, Lemay CG et al. Targeted inflammation during oncolytic virus therapy severely compromises tumor blood flow. Mol. Ther. 15(9), 1686-1693 (2007).

84. Halldén G, Portella G. Oncolytic virotherapy with modified adenoviruses and novel therapeutic targets. Expert Opin. Ther. Targets 16(10), 945-958 (2012).

85. Sorrentino R, Libertini S, Pallante PL et al. Aurora B overexpression associates with the thyroid carcinoma undifferentiated phenotype and is required for thyroid carcinoma cell proliferation. J. Clin. Endocrinol. Metab. 90(2), 928-935 (2005).

86. Lilley CE, Schwartz RA, Weitzman MD. Using or abusing: viruses and the cellular DNA damage response. Trends in Microbiol. 15(3), 19-126 (2007).

87. Nichols GJ, Schaack J, Ornelles DA. Widespread phosphorylation of histone H2AX by species C adenovirus infection requires viral DNA replication. J. Virol. 83(12), 5987-5998 (2009).

88. Touchefeu Y, Vassaux G, Harrington KJ. Oncolytic viruses in radiation oncology. Radiother. Oncology 99(3), 262-270 (2011).

89. Ame JC, Spenlehauer C, de Murcia G. The PARP superfamily. Bioessays 26(8), 882-893 (2004).

90. Davy C, Doorbar J. G2/M cell cycle arrest in the life cycle of viruses. Virology 368(2), 219-226 (2007).

91. McCabe N, Turner NC, Lord CJ et al. Deficiency in the repair of DNA damage by homologous recombination and sensitivity to poly(ADP-ribose)polymerase inhibition. Cancer Res. 66(16), 8109-8115 (2006).

92. Lin SF, Gao SP, Price DL et al. Synergy of a herpes oncolytic virus and paclitaxel for anaplastic thyroid cancer. Clin. Cancer Res. 14(5), 1519-1528 (2008).

93. Schweppe RE, Klopper JP, Korch C et al. Deoxyribonucleic acid profiling analysis of 40 human thyroid cancer cell lines reveals cross-contamination resulting in cell line redundancy and misidentification. J. Clin. Endocrinol. Metab. 93(11), 4331-4341 (2008).

94. Mellman I, Coukos G, Dranoff G. Cancer immunotherapy comes of age. Nature 480(7378), 480-489 (2011).

95. Topalian SL, Taube JM, Anders RA, Pardoll DM. Mechanism-driven biomarkers to guide immune checkpoint blockade in cancer therapy. Nat. Rev. Cancer 16(5), 275-287 (2016).

96. Chen L, Han, X. Anti-PD-1/PD-L1 therapy of human cancer: past, present, and future. J. Clin. Invest. 125(9), 3384-3391 (2015).

97. Wolchok JD, Chiarion-Sileni V, Gonzalez R et al. Overall survival with combined nivolumab and ipilimumab in advanced melanoma. N. Engl. J. Med. 377(14), 1345-1356 (2017).

98. Liu Z, Ravindranathan R, Kalinski P, Guo ZS, Bartlett DL. Rational combination of oncolytic vaccinia virus and PD-L1 blockade works synergistically to enhance therapeutic efficacy. Nat. Commun. 8, 14754 (2017)

-• Demonstrates in cancer models that an oncolytic vaccinia virus attracts effector T cells and induces PD-L1 expression on both cancer and immune cells in the tumor. The dual therapy reduces PD- $\mathrm{L1}^{+}$cells and facilitates nonredundant tumor infiltration of effector $\mathrm{CD8}^{+}$and $\mathrm{CD4}^{+} \mathrm{T}$ cells.

99. Gujar S, Pol JG, Kroemer G. Heating it up: oncolytic viruses make tumors 'hot' and suitable for checkpoint blockade immunotherapies.. Oncoimmunology. e1442169doi: 10.1080/2162402X.2018.1442169 (2018). (Epub ahead of print)

100. Cunha LL, Marcello MA, Ward LS. The role of the inflammatory microenvironment in thyroid carcinogenesis. Endocr. Relat. Cancer 21(3), R85-R103 (2014).

101. Moretti S, Menicali E, Voce P et al. Indoleamine 2,3-dioxygenase 1 (IDO1).is up-regulated in thyroid carcinoma and drives the development of an immunosuppressant tumor microenvironment. J. Clin. Endocrinol. Metab. 99(5), E832-E840 (2014).

102. Page DB, Postow MA, Callahan MK, Allison JP, Wolchok JD. Immune modulation in cancer with antibodies. Annu. Rev. Med. 65, 185-202. (2014). 
103. Chowdhury S, Veyhl J, Jessa F et al. Programmed death-ligand 1 overexpression is a prognostic marker for aggressive papillary thyroid cancer and its variants. Oncotarget 7(22), 32318-32328 (2016).

104. Ahn S, Kim TH, Kim SW et al. Comprehensive screening for PD-L1 expression in thyroid cancer. Endocr. Relat. Cancer 24(2), 97-106 (2017).

105. Kollipara R, Schneider B, Radovich M et al. Exceptional response with immunotherapy in a patient with anaplastic thyroid cancer. Oncologist 22(10), 1149-1151 (2017).

106. Vanden Borre P, McFadden DG, Gunda V et al. The next generation of orthotopic thyroid cancer models: immunocompetent orthotopic mouse models of BRAF V600E-positive papillary and anaplastic thyroid carcinoma. Thyroid 24(4), 705-714 (2014).

107. Champa D, Di Cristofano A. Modeling anaplastic thyroid carcinoma in the mouse. Horm. Cancer 6(1), 37-44 (2015).

108. Reddi HV, Madde P, Cohen YC et al. Antitumor activity of VB-111, a novel antiangiogenic virotherapeutic, in thyroid cancer xenograft mouse models. Genes Cancer 2(10), 993-995 (2011). 
